\title{
Síntese e Caracterização do Copolímero Poli (L-co-D,L Ácido Láctico)
}

\author{
Adriana C. Motta \\ Faculdade de Engenharia Mecânica, Unicamp \\ Eliana A. R. Duek \\ Fac. Engenharia Mecânica, Unicamp \\ Laboratório de Biomateriais, CCMB, PUC
}

\begin{abstract}
Resumo: A aplicação de polímeros biorreabsorvíveis em próteses temporárias é constante nos procedimentos médicos relacionados a fraturas ósseas. Dentre os polímeros bioreabsorvíveis, o poli(L-co-D, L ácido láctico), PLDLA, na relação 70:30, tem sido estudado visando à obtenção de placas e parafusos para a recuperação de traumas nas regiões buco e crâniomaxilofacial. Nessa relação de monômeros obtém-se um polímero amorfo, o que permite uma adaptação do dispositivo ao local do implante durante a cirurgia. Um fator limitante para o uso desse polímero é seu alto custo em função da importação. Neste trabalho o PLDLA foi sintetizado através da polimerização em massa dos monômeros cíclicos do L-ácido láctico e do D, L ácido láctico, utilizando como catalisador o $\mathrm{Sn}(\mathrm{Oct})_{2}$. Obteve-se material de alta massa molar $\left(\mathrm{Mw}=10^{5} \mathrm{~g} / \mathrm{mol}\right)$, o qual foi caracterizado por ${ }^{1} \mathrm{H}$ RMN, ${ }^{13} \mathrm{C}$ RMN, GPC, FTIR e DSC.
\end{abstract}

Palavras-chave: Poli(L-co-D,L ácido lático), síntese, caracterização.

\section{Synthesis and Characterization of the Copolymer Poly(L-co-D,L lactic acid)}

Abstract: Bioreabsorbable polymers are routinely used as temporary prostheses for fractured bones. Among the bioreabsorbable polymers the poly(L-co-D, L lactic acid), PLDLA, in the 70:30 rate has been studied to obtain plates and screws to recuperate traumas in the cranium and maxilla facial regions. In this monomers rate, an amorphous polymer is obtained, which allows adaptation of devices in the local for the implant during the surgery. A limiting factor to use this polymer is its high cost, due to the importation process. In this work PLDLA was synthesized by mass polymerization of the cyclical monomers L-lactic acid and D, L lactic acid, using $\mathrm{Sn}(\mathrm{Oct})_{2}$ as catalyst. A material with high molar mass $\left(10^{5} \mathrm{~g} / \mathrm{mol}\right)$ was obtained and characterized by ${ }^{1} \mathrm{H}$ RMN, ${ }^{13} \mathrm{C}$ RMN, GPC, FTIR and DSC.

Keywords: Poly(L-co-D,L lactic acid), synthesis, characterization.

\section{Introdução}

Dispositivos obtidos a partir de polímeros biorreabsorvíveis aplicados em osteofixação ao têm sido cada vez mais aceitos na área de ortopedia e traumatologia ${ }^{[1]}$. Atualmente, dispositivos como mini placas e parafusos desses polímeros são usados rotineiramente na região crânio e bucomaxilofacial, substituindo os dispositivos metálicos ${ }^{[2]}$.

A utilização de materiais permanentes, como o titânio, na fixação de fraturas já data de muitas décadas. Apesar da elevada propriedade mecânica que tais implantes apresentam, o grande inconveniente de sua utilização reside nos elevados percentuais de remoção destes dispositivos do organismo, após a consolidação da fratura. Um exemplo que descreve a necessidade de remoção dos implantes metálicos no organismo pode ser dado quando o implante é aplica- do a fraturas na região craniofacial em crianças, tendo em vista que a não-remoção do mesmo, implica num comprometimento do crescimento ósseo destes indivíduos, além da possibilidade de migração destes implantes ${ }^{[2-7]}$. Pesquisa recente ${ }^{[8]}$ mostrou que para a maioria dos pacientes, $91 \%$, que possuem algum tipo de implante metálico, o aspecto mais negativo é a necessidade de uma segunda intervenção cirúrgica para retirada do dispositivo. Outro aspecto negativo é a tensão que o metal impõe ao osso neoformado, tendo como resultado um osso com propriedades mecânicas inferiores ao osso normal, com maior propensão a sofrer uma re-fratura num futuro próximo ${ }^{[9]}$.

Entre os polímeros mais utilizados na recuperação de fraturas ósseas, se encontram os poli( $\alpha$-hidróxi ácidos) e seus copolímeros, sendo possível a obtenção de materiais

Autor para correspondência: Adriana C. Motta, Faculdade de Engenharia Mecanica- DEMA, Unicamp, CEP :13083-970, Caixa Postal 6122, Campinas, SP, Brasil.E-mail motta@fem.unicamp.br 
com diferentes propriedades mecânicas, e diferentes taxas de degradação, de acordo com as proporções de monômeros usadas nas sínteses destes copolímeros ${ }^{[10-14]}$. O processo de biorreabsorção desses materiais ocorre através da hidrólise de suas ligações ésteres em contato com os fluidos corpóreos, originando produtos na forma de oligômeros solúveis e não tóxicos, que após sofrerem a ação metabólica do organismo são transformados em $\mathrm{CO}_{2}$ e $\mathrm{H}_{2} \mathrm{O}^{[15]}$.

O poli(ácido láctico) existe em duas formas químicas específicas: poli(L-ácido láctico) e poli(D,L ácido láctico), passíveis de serem combinadas para formar um copolímero com propriedades diferentes daquelas exibidas por cada um desses homopolímeros isolados. Trata-se do copolímero Poli(L-co-D,L ácido láctico), sendo a proporção 70:30 a empregada neste estudo.

A vantagem do copolímero PLDLA é aliar as particularidades mecânicas inerentes ao poli (L-ácido láctico) sem o inconveniente do elevado tempo de degradação requerido por esse homopolímero, decorrente de sua alta cristalinidade. Isso é possível, pois o poli (D,L ácido láctico), ao contrário, apresenta maior taxa de degradação, apesar de possuir propriedades mecânicas aquém das do poli(L-ácido láctico). Com a combinação do L-ácido láctico com o D, L ácido láctico é possível se obter um produto com boas propriedades mecânicas e com tempo de degradação mais adequado às necessidades das aplicações cirúrgicas ${ }^{[16]}$.

A proposta deste estudo foi sintetizar e caracterizar quimicamente o copolímero poli(L-co-D, L ácido láctico) 70:30.

\section{Experimental}

\section{Síntese do copolímero poli(L-co-D,L ácido láctico) - PLDLA}

Copolímeros de L-ácido láctico e D,L ácido láctico (PLDLA) foram preparados através da reação de polimerização em massa, em uma ampola de vidro, por abertura de seus anéis. A proporção entre os monômeros L-lactato (Sigma-Aldrich) e D,L lactato (Sigma-Aldrich) escolhida para as sínteses foi de 70:30, respectivamente. O catalisador utilizado foi o 2-etilhexanoate de estanho II, mais conhecido como $\mathrm{Sn}$ $(\mathrm{Oct})_{2}$, e a relação monômero/catalisador por volta de 5000 . A escolha dessa relação foi baseada nos resultados promissores, de um trabalho anterior envolvendo a síntese do homopolímero PLLA ${ }^{[17]}$. No caso da síntese do copolímero, foco deste trabalho, foi otimizada a relação monômero/catalisador capaz de gerar o PLDLA de alta massa molar através da variação desta relação numa faixa próxima a 5000 ( de 5000 a 7000 vezes a relação). As massas dos monômeros (L-lactato e D,L lactato) e do catalisador foram adicionadas a uma ampola de vidro, a qual foi selada sob vácuo e imersa em um banho de óleo à $130{ }^{\circ} \mathrm{C}$ por 72 horas. Decorrido esse tempo o polímero foi dissolvido em clorofórmio, $\mathrm{CHCl}_{3}$ (Merck) e precipitado em metanol, $\mathrm{CH}_{3} \mathrm{OH}$ (Merck). O material obtido foi seco inicialmente num dessecador e em seguida numa estufa a vácuo numa temperatura de $45^{\circ} \mathrm{C}$ por 8 horas. Todos os copolímeros sintetizados foram pesados e caracterizados pelas seguintes técnicas:

Ressonância magnética (RMN de H1 e C13): Os espectros de RMN foram obtidos em um espectrofotômetro GEMINI 300BB operando a $75 \mathrm{MHz}$ para $\mathrm{RMN}^{13} \mathrm{C}$ e $300 \mathrm{MHz}$ para $\mathrm{RMN}^{1} \mathrm{H}$. Foram utilizados tubos de vidro $5 \mathrm{~mm}$ de diâmetro e clorofórmio deuterado como solvente em temperatura ambiente, numa concentração de $10 \%$ em massa.

Espectroscopia de absorção na região do Infravermelho (FTIR): Os espectros de infravermelho foram obtidos na região de 4000 a $650 \mathrm{~cm}^{-1}$, em amostras na forma de filmes sobre $\mathrm{NaCl}$, em um espectrofotômetro BOMEN MB-Séries.

Cromatografia de Permeação em gel (GPC): As massas molares numérica média (Mn), e ponderal média em massa (Mw) e o índice de polidispersão (IP) foram determinados através de um cromatógrafo de permeação CLWA-1 em gel (GPC) a temperatura $25^{\circ} \mathrm{C}$ utilizando-se THF (tetraidrofurano) como fase móvel, numa concentração de $10 \mathrm{mg} / \mathrm{mL}$. As colunas padrões utilizadas foram as de poliestireno de $10^{2}$, $10^{4}$ e $10^{5} \mathrm{~nm}$, e detector de índice de refração.

Calorimetria Exploratória Diferencial (DSC): Aproximadamente $10 \mathrm{mg}$ das amostras foram aquecidas de 25 a $200{ }^{\circ} \mathrm{C}$, a $5{ }^{\circ} \mathrm{C} /$ min e logo após resfriadas, a $3{ }^{\circ} \mathrm{C} / \mathrm{min}$, até - $50{ }^{\circ} \mathrm{C}$, sendo a seguir aquecidas até $200{ }^{\circ} \mathrm{C}$, a $5{ }^{\circ} \mathrm{C} / \mathrm{min}$, sob purga de $\mathrm{N}_{2}$ em um equipamento Ta Instruments modelo MDSC291.

\section{Resultados e Discussão}

\section{Sínteses do PLDLA}

Para as diversas sínteses de PLDLA realizadas, foram mantidas as condições de temperatura $\left(130^{\circ} \mathrm{C}\right)$ e tempo de síntese (72 horas) assim como a relação monômero/catalisador na faixa de 5000-6500 vezes, como pode ser verificado na Tabela 1, a qual apresenta os resultados de massas molares numérica média $(\mathrm{Mn})$, ponderal média $(\mathrm{Mw})$ e índice de polidispersão (IP) para as diferentes sínteses do PLDLA na relação 70/30, além da relação monômero/catalisador, M/C, empregada em cada síntese, juntamente com o rendimento em massa obtido.

Como pode ser verificado na Tabela 1 , os valores de massa molar, para as sínteses de PLDLA realizadas, foram da ordem de $10^{5} \mathrm{~g} / \mathrm{mol}$, o que caracteriza polímeros de alta massa molar, como é o requerido para as aplicações a que esses materiais se destinam, ou seja, fixadores de fraturas. A Figura 1 mostra as curvas de GPC das amostras de PLDLA.

Verifica-se a estreita relação entre os polímeros (sínteses numeradas como 4, 5 e 8) cuja massa molar se mostrou inferior ao valor de $10^{5} \mathrm{~g} / \mathrm{mol}$ para a massa molar ponderal média $\left(\mathrm{M}_{\mathrm{w}}\right)$ Para esses copolímeros a relação monômero/catalisador se manteve sempre acima de 6500 vezes, indicando que a melhor relação monômero/catalisador, para as condições de sínteses estipuladas, deve ficar na faixa entre 5000 vezes a 
Tabela 1. Massas molares (Mw e Mn) e índice de polidispersão (IP) do PLDLA, rendimento e dados das relações molares entre o monômero proveniente do diéster cíclico do ácido láctico e o catalisador $\mathrm{Sn}(\mathrm{Oct})_{2}$, (M/C) usados nas polimerizações em massa do PLDLA.

\begin{tabular}{lccccc}
\hline \multicolumn{1}{c}{ Síntese } & Razão molar $\mathbf{M} / \mathbf{C}$ & $\mathbf{M w}(\mathbf{g} / \mathbf{m o l})$ & $\mathbf{M n}(\mathbf{g} / \mathbf{m o l})$ & IP (Mw/Mn) & Rendimento (\%) \\
\hline PLDLA-1 & 5330 & 449400 & 279000 & 1,6 & 89 \\
PLDLA-2 & 5010 & 208200 & 121300 & 1,7 & 83 \\
PLDLA-3 & 6428 & 206000 & 120800 & 1,7 & 85 \\
PLDLA-4 & 6518 & 56617 & 31846 & 1,8 & 85 \\
PLDLA-5 & 6555 & 38058 & 24840 & 1,5 & 75 \\
PLDLA-6 & 6387 & 364055 & 219696 & 1,7 & 75 \\
PLDLA-7 & 5935 & 114889 & 74126 & 1,6 & 76 \\
PLDLA-8 & 7000 & 63122 & 39345 & 1,6 & 85 \\
PLDLA-9 & 5111 & 242606 & 138015 & 1,8 & 80 \\
PLDLA-10 & 5284 & 282700 & 131354 & 2,2 & 90 \\
PLDLA-11 & 5254 & 266442 & 158172 & 1,7 & 86 \\
PLDLA-12 & 5305 & 250403 & 145730 & 1,7 & 91 \\
PLDLA-13 & 5257 & 529590 & 257816 & 2,0 & 93 \\
PLDLA-14 & 5265 & 448136 & 229980 & 2,0 & 92 \\
PLDLA-15 & 5225 & 494140 & 298802 & 1,6 & - \\
PLDLA (Purac) comercial & - & 100000 & - & - & 81 \\
\hline
\end{tabular}

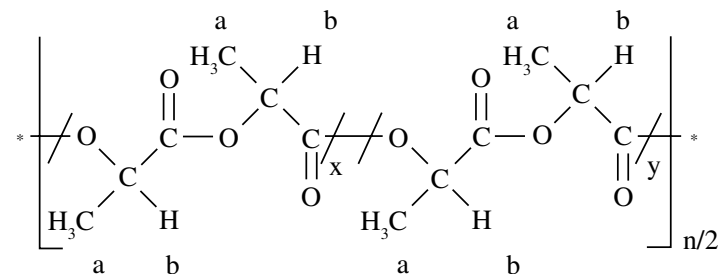

Figura 1. Representação da estrutura do copolímero poli( L-co-D,L ácido lático).

6350 vezes, já que as sínteses cuja relação monômero/catalisador diferiram deste intervalo mostraram valores mais baixos de massa molar.

A busca por valores elevados de massa molar está baseada no fato de que este é um dos parâmetros que influenciarão diretamente na qualidade dos dispositivos poliméricos obtidos, pois valores altos de massa molar produzirão dispositivos com melhores propriedades mecânicas ${ }^{[18]}$.

É bem conhecido que espectros de RMN de polímeros são sensíveis a efeitos de grupos vizinhos, bem como a estruturas configuracionais, sendo assim é fato que o espectro do copolímero em questão, poli(L-co-D, L ácido láctico), se apresente, tanto em relação ao espectro $\mathrm{H}^{1}$ como de $\mathrm{C}^{13}$, mais complexo do que o correspondente ao homopolímero, poli(L ácido láctico). A Figura 1 mostra a estrutura referente ao poli(L-co-D,L ácido láctico), analisada por ${ }^{1} \mathrm{H} \mathrm{RMN}$.

Os sinais encontrados no ${ }^{1} \mathrm{H}$ RMN para o copolímero poli(L-co-D,L ácido lático) estão de acordo tanto com o reportado pela literatura ${ }^{[19]}$ como também o verificado para a amostra referente ao material comercial. Os deslocamentos químicos encontrados foram $\left(\mathrm{CDCl}_{3}, \delta, \mathrm{ppm}\right)$ : no espectro do copolímero, o multipleto em 5,12-5,24 ppm é atribuído aos prótons $\mathrm{CH}$ (b), enquanto o quarteto em 1,55 -1,59 ppm é atribuído aos prótons
$\mathrm{CH}_{3}$ (a). Comparando-se o espectro do copolímero com o do homopolímero, observa-se que, apesar dos deslocamentos em termos de ppm serem muito próximos, o número de desdobramentos de sinais para o copolímero é maior, consequiência das estruturas configuracionais mais complexas neste caso, como pode ser verificado nas Figuras $2 \mathrm{a}$ e $2 \mathrm{~b}$, referentes ao homopolímero poli(L-ácido láctico) e ao copolímero poli(L-co-D,L ácido láctico), respectivamente.

Pode-se verificar claramente que existem mais desdobramentos nos sinais referentes ao copolímero comparado aos verificados no homopolímero. Esses desdobramentos de sinais originam-se da estereosensitividade devido à presença de dois pares enantioméricos do acido láctico no copolímero, que são diastereoisomericamente diferentes, ou seja, pares LL/DD e os LD/DL ${ }^{[20]}$ a Figura 3, compara o copolímero PLDLA-3 com o comercial (Purac).

Pode-se verificar que os espectros se assemelham muito quanto à disposição dos picos (Figuras 3a e b), o que é uma ferramenta a mais na constatação de que o copolímero sintetizado é quimicamente idêntico ao copolímero comercial.

Os espectros das sínteses dos demais copolímeros sintetizados apresentaram picos com os mesmos deslocamentos químicos que o PLDLA-3, portanto não serão mostrados.

Os sinais encontrados no ${ }^{13} \mathrm{C}$ RMN para o copolímero poli (L-co-D,L ácido lático), estão de acordo tanto com o reportado pela literatura, como também o verificado para a amostra referente ao material comercial ${ }^{[21]}$. $\mathrm{O}$ espectro de ${ }^{13} \mathrm{C}$ RMN, Figura 4, mostra os seguintes deslocamentos quími$\cos \left(\mathrm{CDCl}_{3}, \delta, \mathrm{ppm}\right): 16,6\left(\mathrm{C}, \mathrm{CH}_{3}\right) ; 69(\mathrm{C}, \mathrm{CH}) ; 166$ e 169,6 $(\mathrm{C}, \mathrm{C}=\mathrm{O})$ e traz uma comparação entre o espectro de RMN de $\mathrm{C}^{13}$ para o PLDL-3 e o PLDLA comercial (Purac). O sinal a $76,5 \mathrm{ppm}$ refere-se ao solvente. 


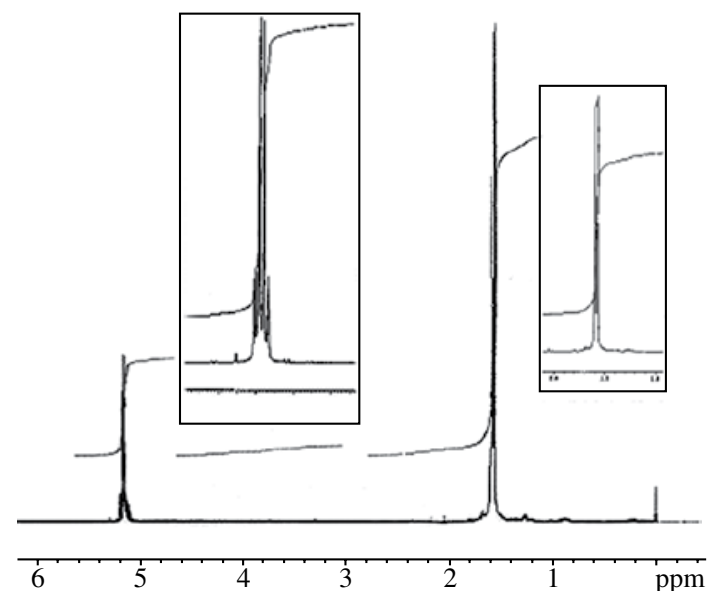

(a)

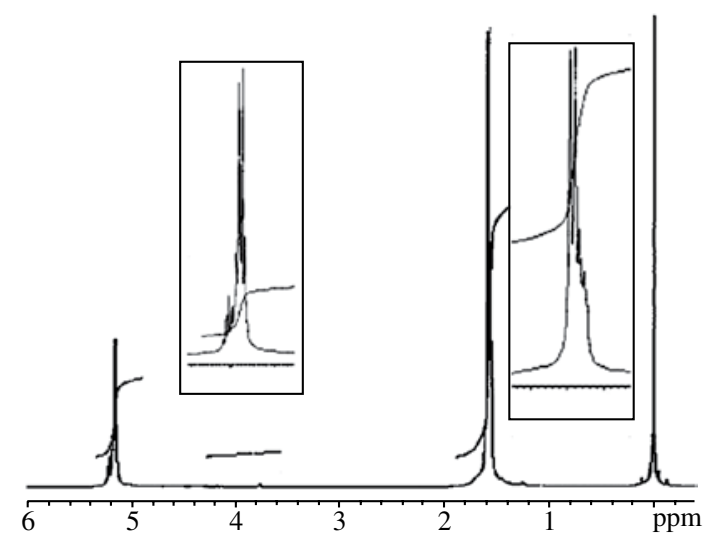

(b)

Figura 2. a) Espectro de ressonância magnética nuclear ${ }^{1} \mathrm{H}$ RMN referente ao PLLA; e b) Espectro de ressonância magnética nuclear ${ }^{1} \mathrm{H}$ RMN referente ao PLDLA-3.

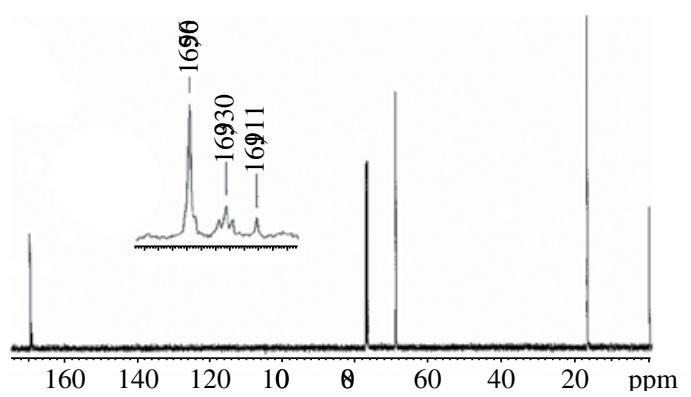

(a)

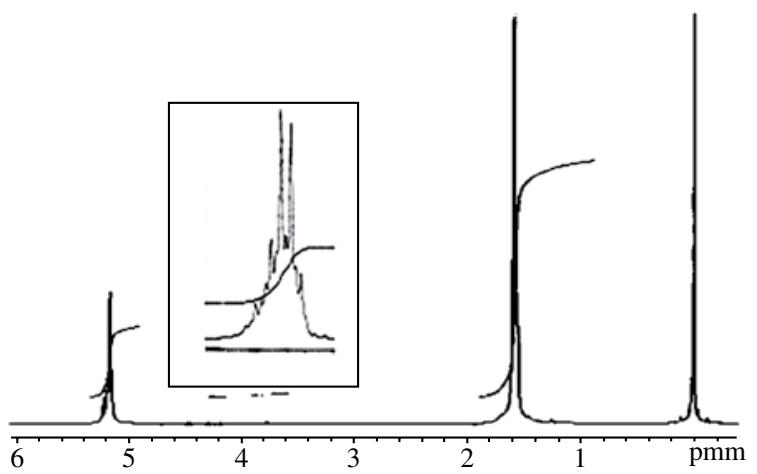

(a)

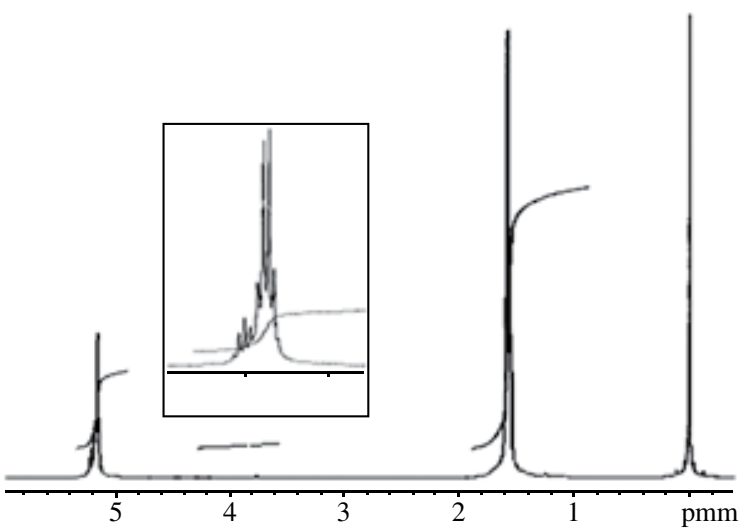

(b)

Figura 3. Espectro de ressonância magnética nuclear de próton ( ${ }^{1} \mathrm{H}$ RMN) referente ao PLDLA, a) todos os sinais verificados no espectro do copolímero comercial (Purac); e b) sinais verificados para o copolímero PLDLA-3, com os mesmos sinais verificados na literatura ${ }^{[17]}$.

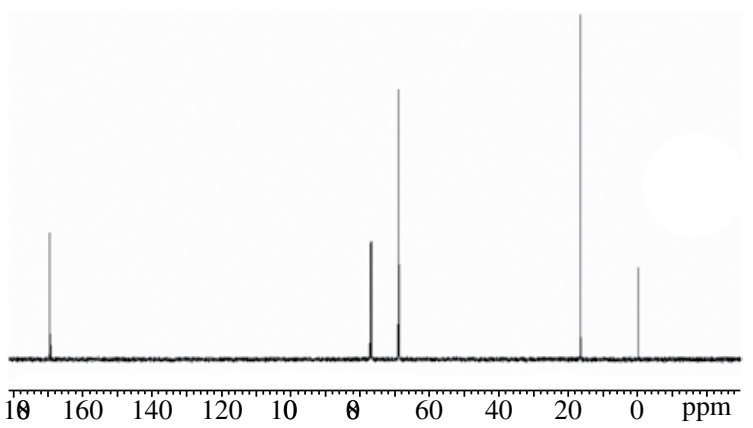

(b)

Figura 4. Espectro de ressonância magnética nuclear $\left(\operatorname{RMN}{ }^{13} \mathrm{C}\right)$ referente ao PLDLA, a) todos os sinais verificados no espectro da amostra PLDLA-3 de acordo com a literatura ${ }^{[21]}$; b) sinais verificados para o copolímero comercial (Purac).

Os espectros das demais sínteses dos copolímeros apresentaram picos com os mesmos deslocamentos químicos que o PLDL-3, portanto não serão mostrados.

Os copolímeros sintetizados foram analisados estruturalmente através de espectroscopia de absorção na região do infravermelho. Todos os copolímeros apresentam as seguintes bandas de absorção $\left(\mathrm{v}, \mathrm{cm}^{-1}\right)$ : 2997-2965 $\left(\mathrm{CH}_{2}, \mathrm{CH}_{3}\right), 1759(\mathrm{C}=\mathrm{O})$, 1360-1450 (CH3), $750(\mathrm{CH})$, que caracterizam o material ${ }^{[22]}$. A Figura 5 mostra uma comparação do espectro referente ao
FTIR do PLDLA com uma amostra comercial do copolímero (Purac), podendo ser notada claramente a similaridade entre as bandas de absorção.

\section{Calorimetria exploratória diferencial (DSC)}

Na Figura 6 estão mostradas curvas de DSC das sínteses dos copolímeros PLDLA podendo ser claramente visualizado a presença da temperatura de transição vítrea, $\left(\mathrm{T}_{\mathrm{g}}\right)$ para todas as amostras, indicando que o material é amorfo. Entretanto, algu- 


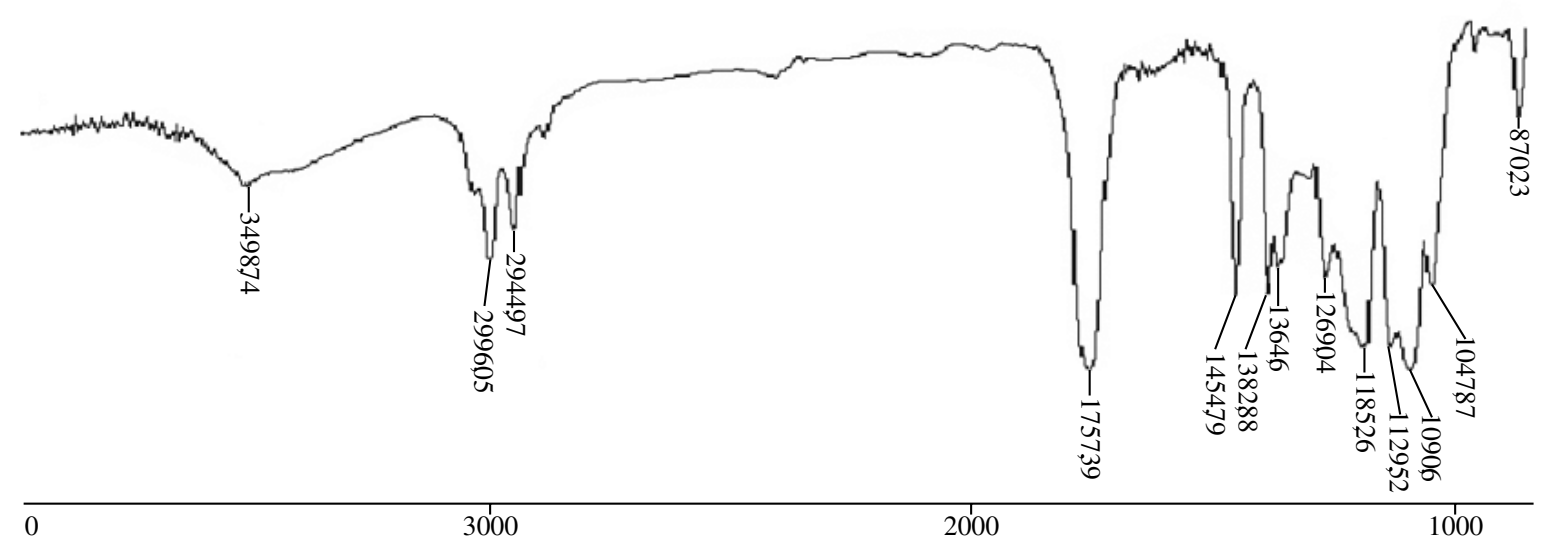

(a)

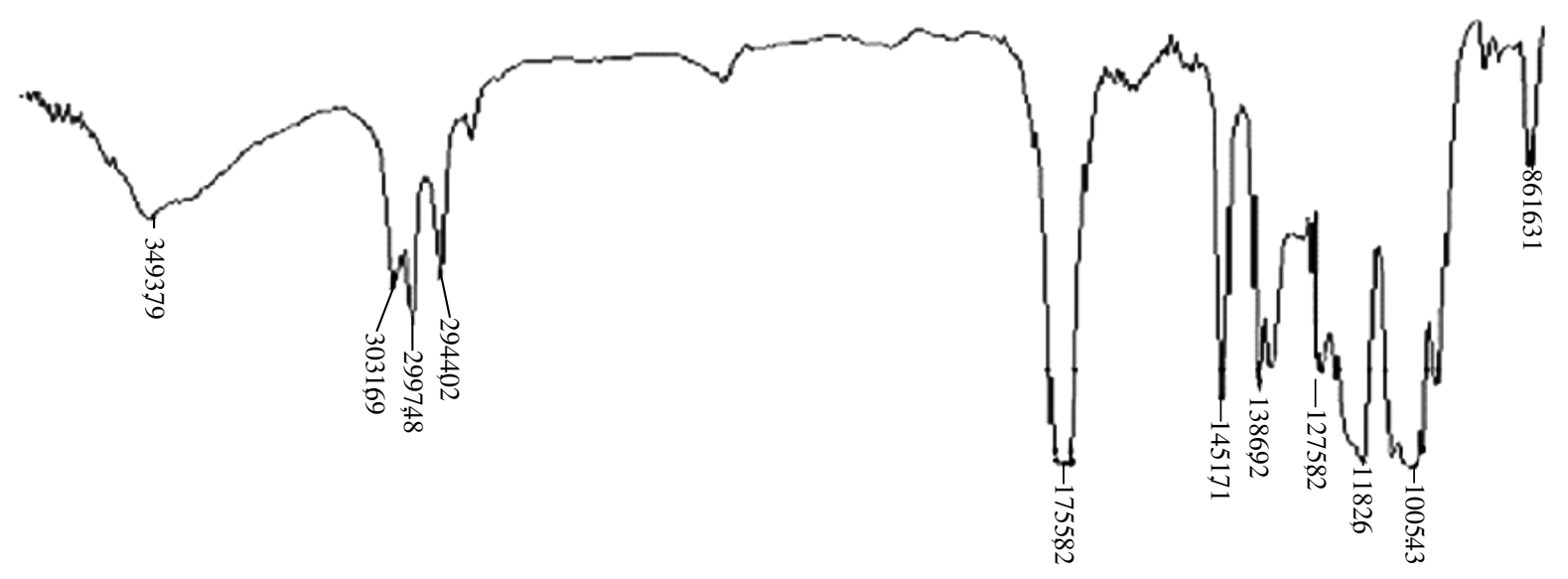

(b)

Figura 5. Comparação entre o espectro na região do infravermelho referente a) copolímero PLDLA-3; e b) comercial (Purac).

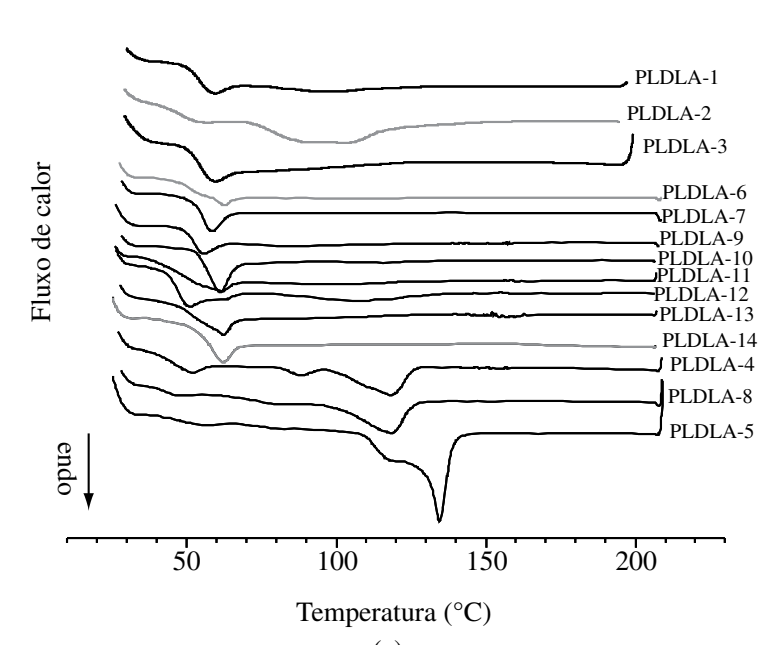

(a)

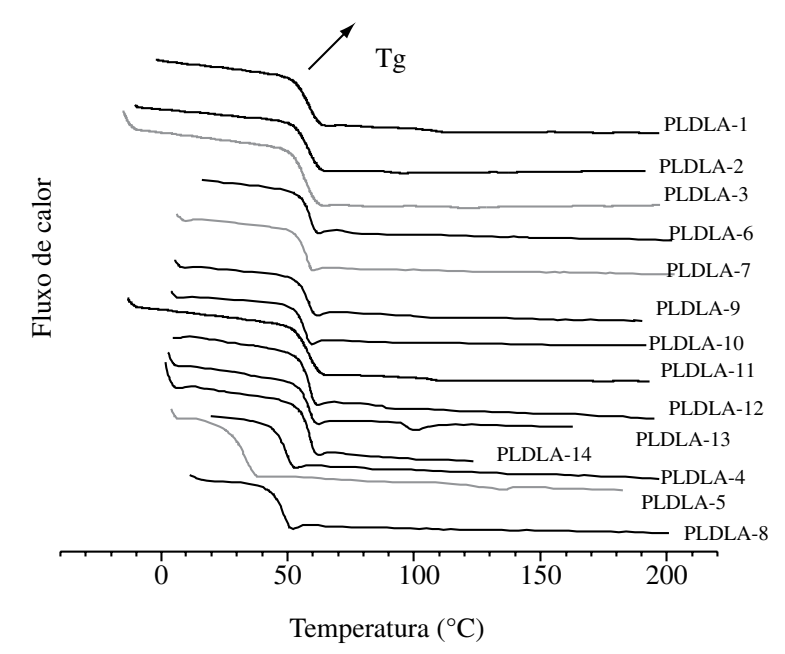

(b)

Figura 6. Curvas DSC das amostras de PLDLA, a) curvas do $1^{\circ}$ aquecimento; e b) curvas do $2^{\circ}$ aquecimento.

mas sínteses (PLDLA-4; PLDLA-5 e PLDLA-8), apresentaram, além da $\mathrm{T}_{\mathrm{g}}$, um discreto pico endotérmico acima de $100{ }^{\circ} \mathrm{C}$. Isso provavelmente tem origem na menor massa mo$\operatorname{lar}(\mathrm{Mw}=56617 \mathrm{~g} / \mathrm{mol}$ para PLDLA 4; $\mathrm{Mw}=38058 \mathrm{~g} / \mathrm{mol}$ para PLDLA-5; Mw = $63122 \mathrm{~g} / \mathrm{mol}$ para PLDLA-8) apresentada por esses copolímeros acima citados, quando comparados às outras sínteses obtidas, já que uma menor massa molar permite um rearranjo das cadeias poliméricas, de forma a originar pequenos cristais ${ }^{[23-25]}$.

O $2^{\circ}$ aquecimento da curva de DSC demonstra que os copolímeros são completamente amorfos, sendo verificado exclusivamente a $\mathrm{T}_{\mathrm{g}}$, em todas as sínteses, cujo valor foi de 
Tabela 2. Propriedades térmicas dos polímeros de PLDLA determinadas via análise DSC, sendo temperatura de transição vítrea $\left(\mathrm{T}_{\mathrm{g}}\right)$; temperatura de fusão $\left(\mathrm{T}_{\mathrm{m}}\right)$; entalpia de fusão $\left(\Delta \mathrm{H}_{\mathrm{m}}\right)$. As analises foram feitas em atmosfera de $\mathrm{He}$, com rampa de aquecimento de $10{ }^{\circ} \mathrm{C} \cdot \mathrm{min}^{-1} \mathrm{e}$ amostras pesando aproximadamente $10 \mathrm{mg}$.

\begin{tabular}{cccc}
\hline Síntese & $\mathbf{T}_{\mathbf{g}}\left({ }^{\circ} \mathbf{C}\right)$ & $\mathbf{T}_{\mathbf{m}}\left({ }^{\circ} \mathbf{C}\right)$ & $\Delta \mathbf{H}_{\mathbf{m}}(\mathbf{J} / \mathbf{g})$ \\
\hline PLDLA-1 & 58 & - & - \\
PLDLA-2 & 58 & - & - \\
PLDLA-3 & 57 & - & - \\
PLDLA-4 & 49 & 118 & 12 \\
PLDLA-5 & 33 & 135 & 23 \\
PLDLA-6 & 59 & - & - \\
PLDLA-7 & 58 & - & - \\
PLDLA-8 & 48 & 118 & 14 \\
PLDLA-9 & 58 & - & - \\
PLDLA-10 & 57 & - & - \\
PLDLA-11 & 58 & - & - \\
PLDLA-12 & 58 & - & - \\
PLDLA-13 & 58 & - & - \\
PLDLA-14 & 59 & - & \\
\hline
\end{tabular}

aproximadamente $58{ }^{\circ} \mathrm{C}$. É justamente o fato do copolímero ser amorfo que torna seu tempo de degradação menor, além de mais flexível, comparado ao homopolímero PLLA, dessa forma mais voltado a aplicações, cujo tempo requerido seja menor.

A Tabela 2 traz os resultados obtidos da $\mathrm{T}_{\mathrm{g}}$ para as amostras, sendo considerados os valores extraídos do $2^{\circ}$ aquecimento, pois a história térmica do material neste aquecimento foi eliminada. Os valores de temperatura de fusão $\left(T_{m}\right)$ e entalpia de fusão $\left(\Delta \mathrm{H}_{\mathrm{m}}\right)$, para as amostras ( PLDL-4; PLDL-5 e PLDL-8) foram obtidos pelo $1^{\circ}$ aquecimento. Como os valores de $\Delta \mathrm{H}_{\mathrm{m}}$ verificados são muito baixos, pode-se assegurar que se trata somente de vestígios de cristalinidade, sendo notado que, entre essas sínteses é justamente a de menor massa molar (PLDLA-5 Mw $=38058 \mathrm{~g} / \mathrm{mol}$ ) que teve o maior valor de $\Delta \mathrm{H}_{\mathrm{m}}$, sendo uma explicação provável para isso o rearranjo mais facilitado encontrado por essas cadeias poliméricas, o que torna esses cristais com menos imperfeições, portanto com um valor de $\Delta \mathrm{H}_{\mathrm{m}}$ maior quando comparadas as demais ( PLDL-4 e PLDL-8).

\section{Conclusões}

Os resultados obtidos, para a maior parte das sínteses de poli(L-co-D,L ácido lático) 70:30 realizadas, mostraram a obtenção de copolímeros de alta massa molar, $\left(10^{5} \mathrm{~g} / \mathrm{mol}\right)$, assim como as propriedades térmicas necessárias para aplicação de próteses temporárias. Foi verificado que quando a polimerização do PLDLA possuía uma relação monômero/catalisador acima de 6500 vezes (o que ocorreu com as sínteses numeradas como PLDLA-4; PLDLA-5 e PLDLA-8) o copolímero obtido apresentava um valor de massa molar inferior àquele possível para copolímeros obtidos numa con- dição em que a relação monômero/catalisador se mantinha entre 5300-6350 vezes.

A caracterização química, através das técnicas de ${ }^{1} \mathrm{H}$ RMN e ${ }^{13} \mathrm{C}$ RMN e FTIR apresentaram resultados de acordo com o relatado na literatura. Também se mostraram de acordo com a literatura as análises térmicas do PLDLA através da técnica de DSC sendo verificado a presença exclusiva da temperatura de transição vítrea $\left(\mathrm{T}_{\mathrm{g}}\right)$, que caracteriza um material amorfo, salvo as sínteses PLDLA-4; PLDLA-5 e PLDLA-8, que apresentaram além da $\mathrm{T}_{\mathrm{g}}$ um pequeno pico endotérmico $\left(\mathrm{T}_{\mathrm{m}}\right)$, em função da relação monômero/catalisador estar acima da adequada nestes casos, acarretando valores de massa molar menores, o que possivelmente levou a um rearranjo da cadeia polimérica e formação de pequenos cristais.

\section{Referências Bibliográficas}

1. Prokop, A.; Jubel, A.; Helling, H. J. \& Eibach, C. - Biomaterials, 25, p.259 (2004).

2. Cohen, S. R.; Holmes, R. E.; Amis, P.; Fitchner, H. \& Shusterman, E. - J. Craniofac. Surg., 12, p.596-602 (2001).

3. Fearon, J. A.; Munro, I. R. \& Bruce, D. - Plast. Reconstr. Surg., 95, p.634-7; discussion 638 (1995).

4. Goldberg, D. S.; Bartlett, S; Yu, J. C.; Hunter, J. V. \& Whitaker, L. A. - J. Craniofac. Surg., 6, p.301-7; discussion 308 (1995).

5. Honig, J. F.; Merten, H. A. \& Luhr, H. - J. Craniofac. Surg., 6, p.292-8; p.299-300 (1995).

6. Stendel, R.; Krischek, B. \& Pietila, T. - Acta Neurochir. (Wien), 143, p.237-43 (2001).

7. Yu, J. C.; Bartlett, S. P.; Goldberg, D. S.; Gannon, F.; Hunter, J.; Habecker, P. \& hitaker, L. - J. Craniofac. Surg., 7, p.64-8 (1996).

8. Mittal, R.; Morley, J; Dinopoulos, H.; Drakoulakis, E.; Vermani, E. \& Giannoudis, P. - Injury. Int. J. Care Injured, 36, p.333-338 (2005).

9. Brodke, D. S.; Gollogly, S; Alexander, M. R; Nguyen, B. K.; Dailey, A. T.; \& Bachus, K. - Spine, 26, p.1324-9 (2001).

10. Gogolewski, S.; Pineda, L. \& Bu“C. M. - Biomaterials, 21, p.2513-20 (2000).

11. Mainil-Varlet, P.; Rahn, B. A. \& Gogolewski, S. - Biomaterials, 18, p.257-66 (1997).

12. Gugala, Z. \& Gogolewski, S. - J. Orthop. Trauma, 13, p.187-95 (1999).

13. Leiggener, C. S.; Curtis, R.; Puger, D.; \& Rahn, B. A. - J. Biomed Mater. Res., 61, p.131-7 (2002).

14. Amano, Y.; Sekiguchi, K.; Shibukawa, Y. \& Yamada, S. - Oral Surg. Oral, 97, p.155-63, (2004). 
15. Huang, M. H.; Li, S.; Hutmacher, D. W.; Schantz, J. T.; Vacanti, C. A.; Braud, C. \& Vert, M. - J. Biomed. Mater. Res, 69A, p.417 (2004).

16. Hu, Y.; Zhang, Z.; Song, D. \& Wang, Y. - Chinese Chemical Letters, 11, p.1023-1026 (2000).

17. Motta, A. C. \& Duek E. A. R. - Polímeros, 16 p.26 (2006).

18. Gogolewski, S. - Injury Int. J. Care Injured, 31, p.S-D28-32 (2000).

19. Thakur, K; Kean, R; Hall, E; Kolstad, J; Lindgren, T; Doscotch, M; Siepmann, J. \& Munson E. - Macromolecules, 30, p.2422 (1997).

20. Espartero, I.; Rashkov, S; Li, N. \& Manolova, M. Vert- Macromolecules, 29, p.3535-3539 (1996).
21. Kister, G.; Cassaras, G. \& Vert, M. - Polymer, 39, p.267-73 (1998).

22. Menem, K. \& Diniz, G. - Turk J. Chem., 13, p.153-161 (1999).

23. Duek, A. R.; Zavaglia, C. A. C. \& Belangero, W. D. Polymer, 40, p.6465-73 (1999).

24. Tsuji, H. \& Ikada, Y. - Polym. Degrad. and Stab, 67, p.179 (2000).

25. Tsuji, H. \& Ikada, Y. - J. Appl. Polym. Sci., 631, p.855 (1997).

Enviado: 06/10/06 Reenviado: 29/01/07

Aceito: 16/02/07 